\title{
Proliferation index and karyometric features of pancreatic intraductal proliferative lesions
}

\author{
Romana Tomaszewska ${ }^{\mathrm{a}}$, Krzysztof Okoń ${ }^{\mathrm{a}}$, \\ Krystyna Nowak ${ }^{\mathrm{b}}$ and Jerzy Stachura ${ }^{\mathrm{a}, *}$ \\ ${ }^{a}$ Department of Pathomorphology, Collegium \\ Medicum, Jagiellonian University, Kraków, Poland \\ ${ }^{\mathrm{b}}$ Department of Surgery, Collegium Medicum, \\ Jagiellonian University, Kraków, Poland
}

\begin{abstract}
The increasing frequency and poor prognosis in pancreatic cancer prompt us to search for morphological lesions being a substrate for its development. Studies of autopsy and surgically resected material as well as recent molecular studies have proved that one of the possible pathways of pancreatic neoplasia is the intraepithelial proliferation - dysplasia - cancer sequence. In the present paper we studied the proliferative activity (Ki-67 index) in pancreatic intraepithelial proliferative lesions and its correlation with geometric features of cell nuclei as signs of increasing dysplasia. The studies were carried out in a group of 35 patients operated on for pancreatic cancer, chronic pancreatitis and other conditions not associated with the pancreas. We used immunohistochemical methods and basic morphometric parameters. The results of our studies indicate that the cell proliferative activity depends both on the type of epithelial proliferation and underlying pancreatic disease. The values of Ki-67 index are significantly different in low-grade proliferation (flat and papillary hyperplasia) and high-grade proliferation (atypical papillary hyperplasia and carcinoma in situ). A set of karyometric features correlates with $\mathrm{Ki}-67$ index but there is no single feature which would have a diagnostic value.
\end{abstract}

Keywords: Pancreas, incipient neoplasia, classification, Ki67 , image analysis

\section{Introduction}

Ductal carcinoma of the pancreas belongs to a group of neoplasms which are characterized by increasing morbidity and mortality and is associated with a very

\footnotetext{
*Correspondence to: Jerzy Stachura, MD, Department of Pathomorphology, Collegium Medicum, Jagiellonian University, ul. Grzegórzecka 16, 31-531 Kraków, Poland. Tel.: +4812 4211564; Fax: +4812 4215210; E-mail: mpstachu@cyf-kr.edu.pl.
}

short survival from the time of its diagnosis. As in most neoplasms, oncogenesis in the pancreas is a multistep process which includes progression from intraepithelial proliferation with dysplasia to carcinoma in situ and in consequence to infiltrating carcinoma. Pancreatic intraepithelial proliferative lesions have long been regarded as the morphological substrate of precancerous lesions in studies of autopsy and surgically resected material, which has been confirmed by molecular studies on the expression of such oncogenes as K-ras and c-erbB-2 and inactivation of tumor suppressor gene p53 [8,13,17,18,20,22,39]. In view of the extremely poor prognosis in pancreatic cancer and its increasing frequency it is very important to establish a method for its early diagnosis. Ultrasonography guided fine needle aspiration biopsy has become a routine diagnostic procedure to identify pancreatic lesions. One should be aware of the fact that biopsy samples contain elements from intraepithelial proliferation areas. The knowledge of morphological features in individual types of proliferation and their role in pancreatic neoplasia will permit identification of patients from groups at risk for the development of pancreatic cancer.

Classification of pancreatic epithelial intraductal proliferation is based on histological and cytological features. When dividing the proliferative lesions into individual groups we take into account such features as cell size, nuclear shape, its location with respect to the cell base, nuclear membrane contour, chromatin arrangement and nucleolar size. The histological features include the formation of papillary projections or the so-called bridging. Dysplastic changes in proliferating epithelium of pancreatic ducts are assessed according to the classical criteria, which is always influenced by certain subjective factors. For this reason the borders between individual types of pancreatic intraepithelial proliferative lesions are "flexible" and part of the cases may be misdiagnosed. From the practical viewpoint it is most important to find such a feature which will demarcate between "reactive" proliferations and those with a tendency towards progression to cancer. One of the characteristic features of neoplasms is an increase 
in the number of cells. A classical method for measuring the proliferative activity is to count mitotic figures, which according to most investigators are associated with certain errors and dependent on many variables for instance tissue fixation. At present in the assessment of proliferative activity we use a monoclonal antibody Ki-67 reacting with non-histone nuclear protein 395 and $345 \mathrm{kD}$ encoded by a single gene on chromosome 10 , whose expression is closely related to the cell cycle. When a resting cell transforms into a proliferating cell the antigen is expressed in the nucleus, whereas it is not detectable in the resting cell $[9,10]$. Its expression appears in phase G1, increases during further stages of the cell cycle, and after cell division abruptly decreases. Assessment of the cell proliferative activity using Ki-67 has been used in cancer of the bronchus, larynx, breast and dysplastic epithelial lesions in Barrett's esophagus [11,16,24,31]. It is believed that Ki-67 index may have an auxiliary value in defining the degree of epithelial dysplasia. The monoclonal antibody MIB-1 is especially frequently applied to study fixed and paraffin embedded material [5, 25].

The purpose of the present study was to evaluate Ki-67 index in pancreatic intraepithelial proliferative lesions as a sign of increasing epithelial proliferation and dysplasia. Additionally, because until now there have been no morphometric studies relating karyometric features to individual types of proliferative lesions, we made an attempt to apply morphometry in demarcating between intraepithelial proliferations of "reactive" type and proliferations with a tendency towards progression to cancer.

\section{Material and methods}

The study population consisted of 35 patients undergoing pancreatectomy or diagnostic sampling of the pancreas due to infiltrating pancreatic cancer (group I14 patients; 8 men, mean age 59.6 years, and 6 women, mean age 59.3 years), chronic pancreatitis (group II 11 patients, 6 men, mean age 51 years, and 5 women, mean age 62 years), and 10 patients operated on for carcinoma of the papilla of Vater (6 cases), gastric carcinoma ( 2 cases), common bile duct cancer, and lymphoma ( 1 case each). In group III there were 4 men (mean age 65.6 years) and 6 women (mean age 63.5 years). In all groups tissue for histopathological examination was obtained from pancreatic parenchyma (in group I beyond macroscopic infiltrating carcinoma).
The tissue was fixed in formalin embedded in paraffin and stained with hematoxylin and eosin. In group I in 2 cases in postoperative material no normal pancreatic parenchyma was found beyond infiltrating cancer. Histologically we evaluated pancreatic intraepithelial proliferative lesions and histological type and grade of pancreatic infiltrating cancer. Types of intraepithelial proliferation were classified according to the criteria defined by Kozuka, and Cubilla and Fitzgerald [7, 21]:

- flat mucosal hyperplasia (FH) defined as a uniform increase in the mucin content of the epithelial cells resulting in a tall columnar mucin producing epithelium;

- papillary hyperplasia $(\mathrm{PH})$ referring to the presence of papillae lined by columnar cells in the ducts and ductules without atypia;

- atypical papillary hyperplasia (APH) indicating papillary lesions with significant cytological atypia (nuclear enlargement, increased nuclear/ cytoplasmic ratio, loss of polarity and nuclear pleomorphism);

- carcinoma in situ (CIS) defined as atypical hyperplasia with tall columnar epithelium having papillary projections without a fibrous core, bridging of papillary projections and significant cytological alterations similar to those in invasive carcinoma.

For the immunolocalisation of Ki-67 sections were stained with the mouse monoclonal antibody Ki-67 Antigen MIB-1 Ready-to-use (Immunotech, France) according to DAKO Optimized Staining System Microwaving Preparation using TechMate Horizon manufactured by LJL Biosystem Inc. (BSA modified method). The slides were immersed in boiling citrate buffer ( $\mathrm{pH} \mathrm{6)}$ in a microwave oven with two changes of buffer for 5 minutes each. Sections were counterstained in Harris hematoxylin and mounted with DAKO glycergel.

Initial assessment of the proliferative activity in epithelial cells of the ducts was done under light microscopy. Only visible, granular nuclear reaction, brownish in colour was regarded as positive reaction. Reactive lymph nodes were used as positive control. In part of the cases, lymphocytes in the pancreatic parenchyma served as internal control.

Image acquisition and analysis hardware consisted of Axioscop microscope (Zeiss) with a Plan-NeoFluar lens set. The ZVS-47DE color CCD camera (manufactured by Optronics, USA) was connected to Gra- 
BIT PCI card (manufactured by Soft Imaging Systems GmbH, Germany) of a PC using RGB line.

Proliferation index was measured by the method previously described [29]. Briefly, images were taken with the CCD camera mounted on an Axioscop Microscope (Zeiss) with a Plan-NeoFluar $40 \times$ lens. To maintain identical parameters of the acquisition the white balance and exposure time was set automatically for each measurement session. Our own applications written in Imaging C (ANSI C) working in the image analysis system AnalySIS pro 2.11 (Soft Imaging System GmbH, Germany) were used [29,44].

Immunopositive cells were counted in fields showing only the proliferative lesion of choice. Fields with a strong background were rejected. After image acquisition the background was corrected using a reference image. The software permitted interactive adjustment of the background segmentation thresholds according to image brightness. In each case we evaluated about 1000 cell nuclei, defining the percentage of nuclei showing strong immunopositivity.

For karyometry purpose, image acquisition was performed with the same system, with the CCD camera mounted on an Axioscop Microscope (Zeiss) with a Plan-NeoFluar $100 \times$ oil immersion lens. The software was running under AnalySIS 2.11 pro image analysis system in Windows 95 environment, on standard PC compatible computer. All programs were developed on purpose in Imaging C (ANSI C compatible) language, using AnalySIS function library, by one of us (K. O.) $[1,2,27,30,33,34,45]$.

For improved performance, the processing was separated into two phases. First, in acquisition and preprocessing phase, images of individual nuclei were prepared. On the slide, region containing searched lesions were marked by one of us (R. T.). Images were introduced into imaging system, following a systematic rule, for avoiding storing the same nuclei twice. Prefiltered image was thresholded by automatic method, with possible interaction by operator. Well-segmented nuclei were then selected, with manual correction of the outline, if needed. Obtained images, containing nuclei on black background were stored in packed-bit compressed TIFF format on magnetooptical disk. Each file contained also tags with codes for slide number, experimental group, and morphological lesion.

The processing phase was fully automatic. All images on magnetooptical disk were serially loaded into operating memory, and surface area, minimum, mean and maximum diameter measured. Shape features used were four form factors defined as follows

$$
\begin{aligned}
& \mathrm{SF}=\frac{4 \pi S}{L^{2}}, \quad R_{\mathrm{f}}=\frac{L_{\mathrm{h}}}{L_{\mathrm{v}}}, \quad R_{\mathrm{c}}=\frac{2 \sqrt{S / \pi}}{L / \pi}, \\
& \text { Elongation }=\frac{D_{\min }}{D_{\max }},
\end{aligned}
$$

where $L$ is nuclear perimeter, and $S$ is nuclear area. $L_{\mathrm{h}}$ and $L_{\mathrm{v}}$ are horizontal and vertical diameters, $D_{\min }$ and $D_{\max }$ maximum and minimum diameter, respectively [37].

Features of each nucleus, together with data from TIFF file tags, were stored in a text file.

For data analysis Microsoft Excel 4.0 (Microsoft Corp.) and STATISTICA for Windows 5.1 (StatSoft, Inc.) were used. Kruskal-Wallis ANOVA, MannWhitney $U$-test and Spearman rank correlation coefficient were calculated. The statistics were deemed significant at $p<0.05$.

\section{Results}

\subsection{Histopathological study}

In group I (pancreatic cancer) in 2 cases in samples obtained for histopathological study there was no pancreatic parenchyma outside tumor area. Additionally in one case in samples obtained from macroscopically normal parenchyma we also found cancer infiltration. In all cases these were intermediate-grade adenocarcinomas. In 4 cases we found foci of carcinoma in situ in pancreatic parenchyma beyond cancer infiltration. The epithelium of ducts in 7 cases showed the signs of atypical papillary hyperplasia, in 8 cases papillary hyperplasia without atypia and in 9 cases flat hyperplasia. In 9 cases part of the ducts was lined with normal epithelium.

In group II (pancreatitis) only in one case we found papillary hyperplasia without atypia and in 5 cases flat hyperplasia. Most ducts were lined with normal epithelium.

In group III (extrapancreatic neoplasms) in 4 cases we found papillary hyperplasia without atypia and in 5 cases flat hyperplasia. Most ducts were lined with normal epithelium.

In groups II and III in none of the cases atypical papillary hyperplasia or foci of carcinoma in situ were found. 


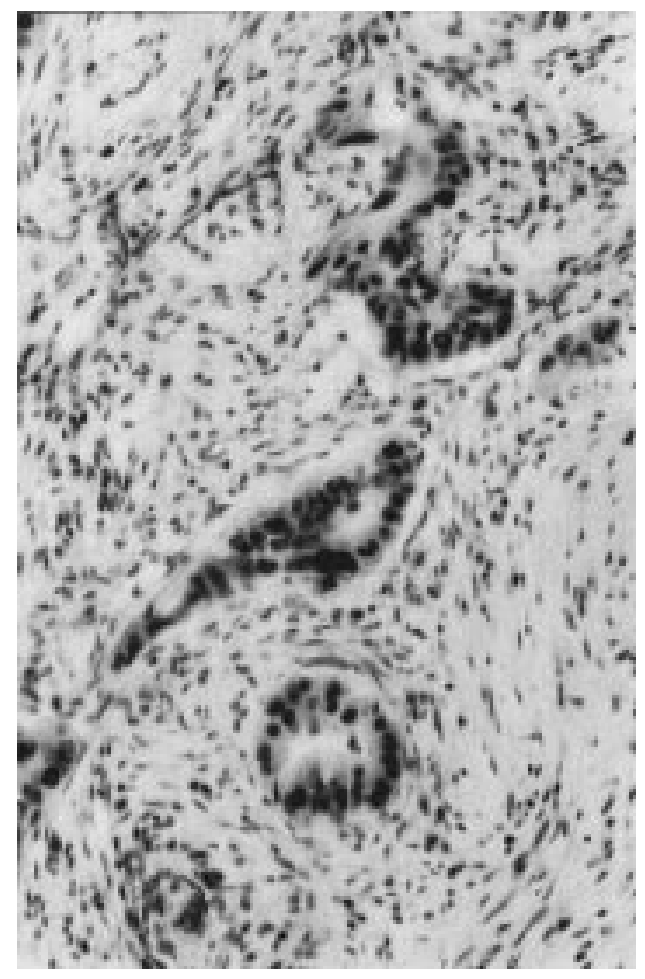

(B)

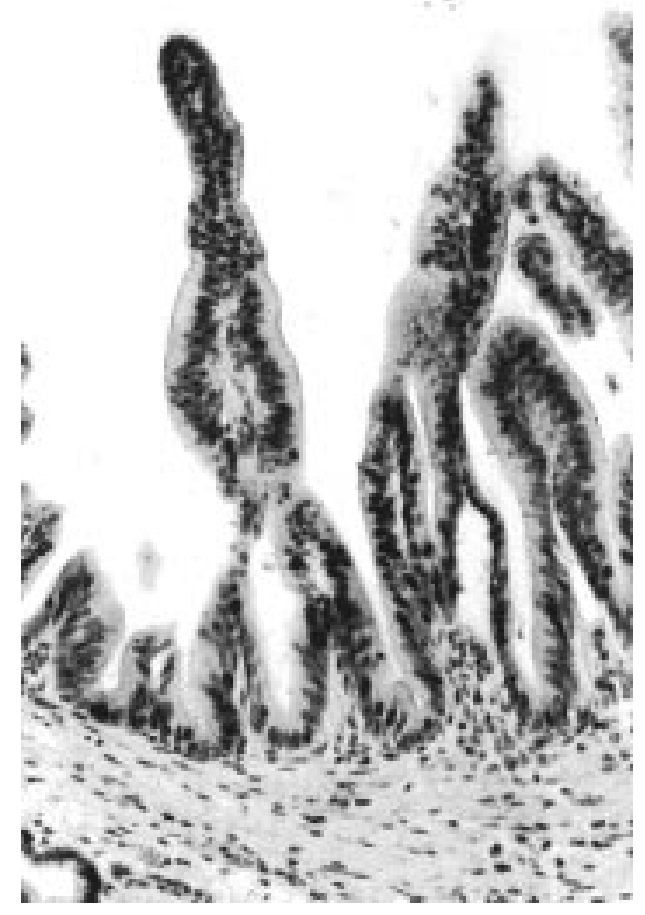

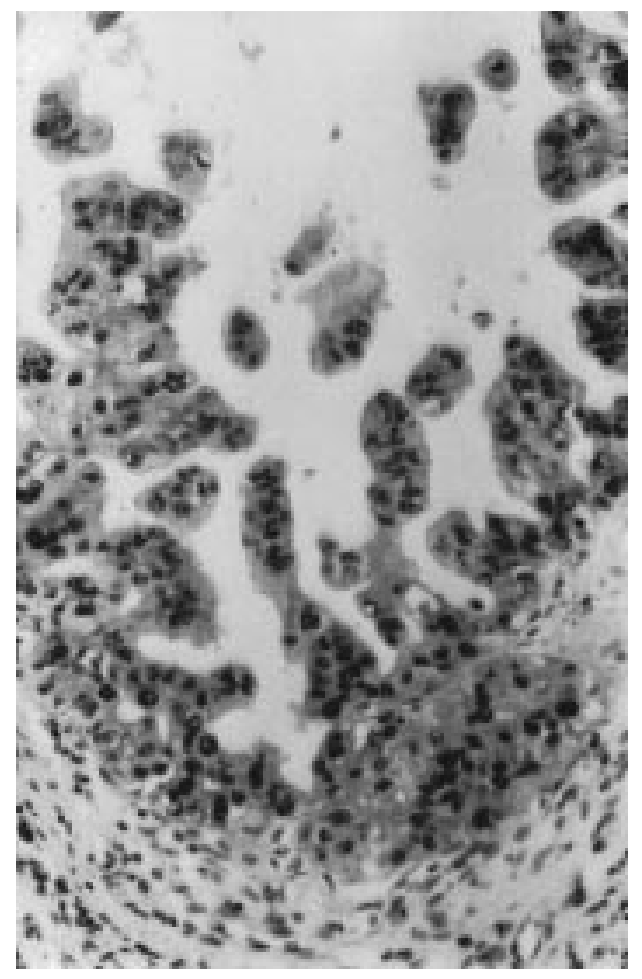

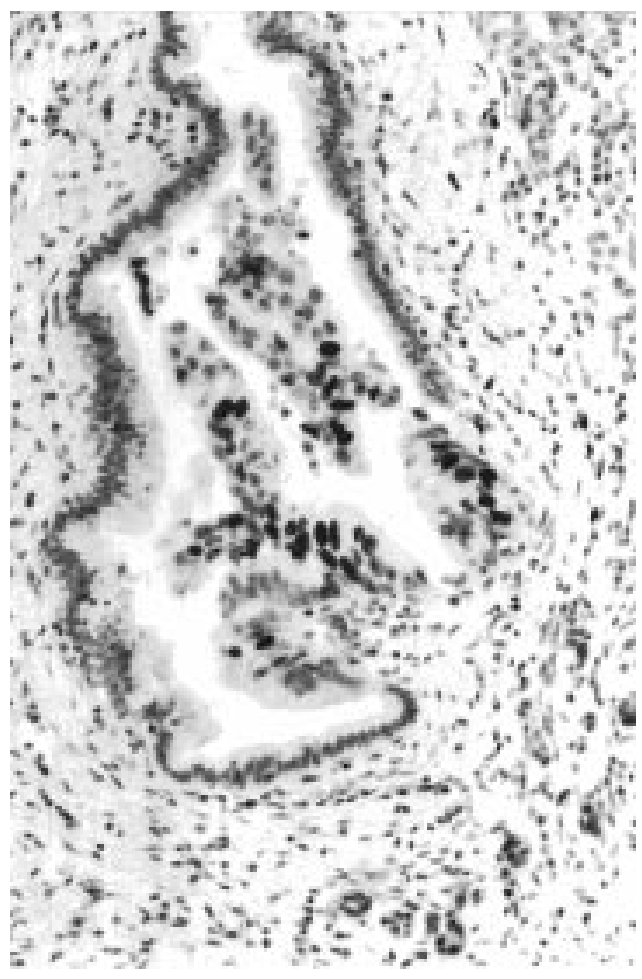

Fig. 1. Immunohistochemical staining for Ki-67. (A) Pancreatic infiltrating carcinoma: almost all cells show positive reaction (165×). (B) Pancreatic in situ carcinoma $(300 \times)$. (C) Atypical papillary hyperplasia. Most cells in the papillary projections show positive staining (165×). (D) Foci of atypical papillary hyperplasia show positive staining in contrast to negative cells in flat hyperplasia $(250 \times)$. 


\subsection{Proliferation index}

The MIB-1 expression was shown in almost all cases, the negative cases were found only in lowgrade lesions and only in the non-neoplastic population (group II and III) (Fig. 1). When comparing the proliferative index in individual types of histological changes between study groups we found the highest values in group I, whereas lowest in group II. In group I the proliferative index gradually increased reaching the maximum in infiltrating cancer. In normal ductal epithelium and in flat and papillary hyperplasia without atypia Ki-67 index showed different values in various study groups (Fig. 2).

\subsection{Geometric features of the nuclei}

By measuring geometric features and form factors of the selected nuclei a variable distribution in different groups could be seen (Fig. 2). The size of nuclei, expressed as means of surface area, as well as minimum and maximum diameter, increased in normal $\rightarrow$ hyperplasia $\rightarrow$ carcinoma sequence, and variation of these parameters increased also, with a drop for in situ carcinoma. The same effects were seen in each of the experimental groups, at the extent consistent with the lesions present in them; these increases were most evident for group I (pancreatic cancer), and only slight for group II (pancreatitis), being intermediate for group III (nonpancreatic cancer). In fact, on Kruskal-Wallis ANOVA these differences are highly significant in group I, and III $(p<0.015)$, and not significant in group II. These findings indicate, that nuclear enlargement is present in ductal epithelium around pancreatic carcinoma histologically classified at low end of the spectrum of pancreatic intraductal proliferative lesions, a phenomenon not present in tissue from pancreatitis cases. In respect to shape of the nuclei, represented by the set of form factors presented in the Material and methods Section, it became more oval in the $\mathrm{PH}$ group, approximating to circular in both ends of the spectrum. Significant differences between the lesions could be shown for some, but not all parameters in all experimental groups.

In agreement with our previous experiences [39], we decided to group the proliferative lesions into low and high grade groups. Low grade consisted of normal, flat hyperplasia and papillary hyperplasia, and high grade of atypical papillary hyperplasia, carcinoma in situ and infiltrating carcinoma. The rationale for doing this is that so defined high grade lesions are specific for pancreatic carcinoma patients. When comparing individ- ual lesions classified into low grade class, most parameters did not show statistically significant differences; a similar phenomenon may be observed in lesions classified into high grade class. In contrast, when comparing low and high grade groups significant differences are noted (Table 1).

When comparing individual low grade lesions between study groups, differences between them are seen, some of them are statistically significant. Therefore lesions with identical morphological classification differ in various groups. It indicates that even relatively unchanged ductal epithelium obtained from the vicinity of pancreatic cancer shows differences with respect to unchanged (or slightly changed) epithelium from control groups. It is evident when examining proliferation index in the ductal epithelium deemed normal by optical microscopy: in non-neoplastic category no Ki67 positive cells are identified, whereas in pancreatic carcinoma-bearing patients they are slightly increased in number. Our findings suggest altered nuclear parameters of ductal cells in pancreatic ductal carcinomabearing patient and indicate early events in carcinogenesis.

Proliferation index is correlated with geometric parameters in Table 2. The MIB-1 index is significantly correlated with these parameters, but this effect tends to disappear when the data set is limited to individual lesions (not shown) [23,36,45].

\section{Discussion}

A constant increase in the number of cells within a neoplasm is one of the most typical features, and the proliferative activity is an important prognostic factor. A commonly used technique of studying proliferation is counting the number of mitotic figures. However, this technique is associated with low reproducibility and sensitivity to artifacts. Furthermore, "mitosis", i.e., a figure of division is only a fragment of the cell cycle and treating the number of mitoses as an index of proliferation markedly reduces a fraction of proliferating cells. However, not all cells in $\mathrm{S}$ phase must divide, which means that only the number of mitoses defines a fraction of truly dividing cells. Mitotic figures are very sensitive for instance to delayed fixation, especially in tumors with high mitotic activity. Another method of study is labeled thymidine incorporation, but it is time consuming and thus not suitable for routine diagnosis. In contrast, Ki-67 index seems the best marker to study the rate of proliferation, especially that time of 

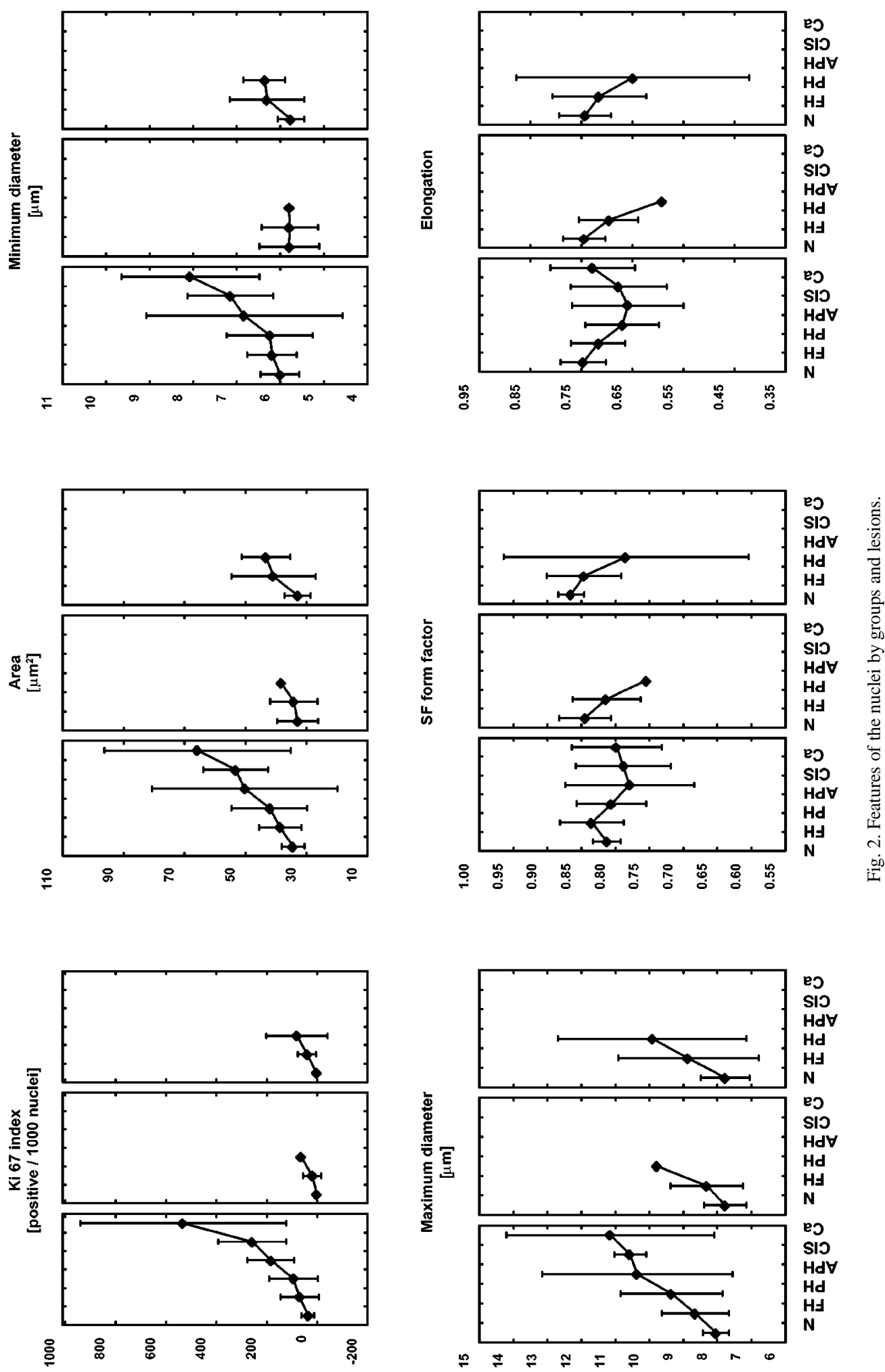

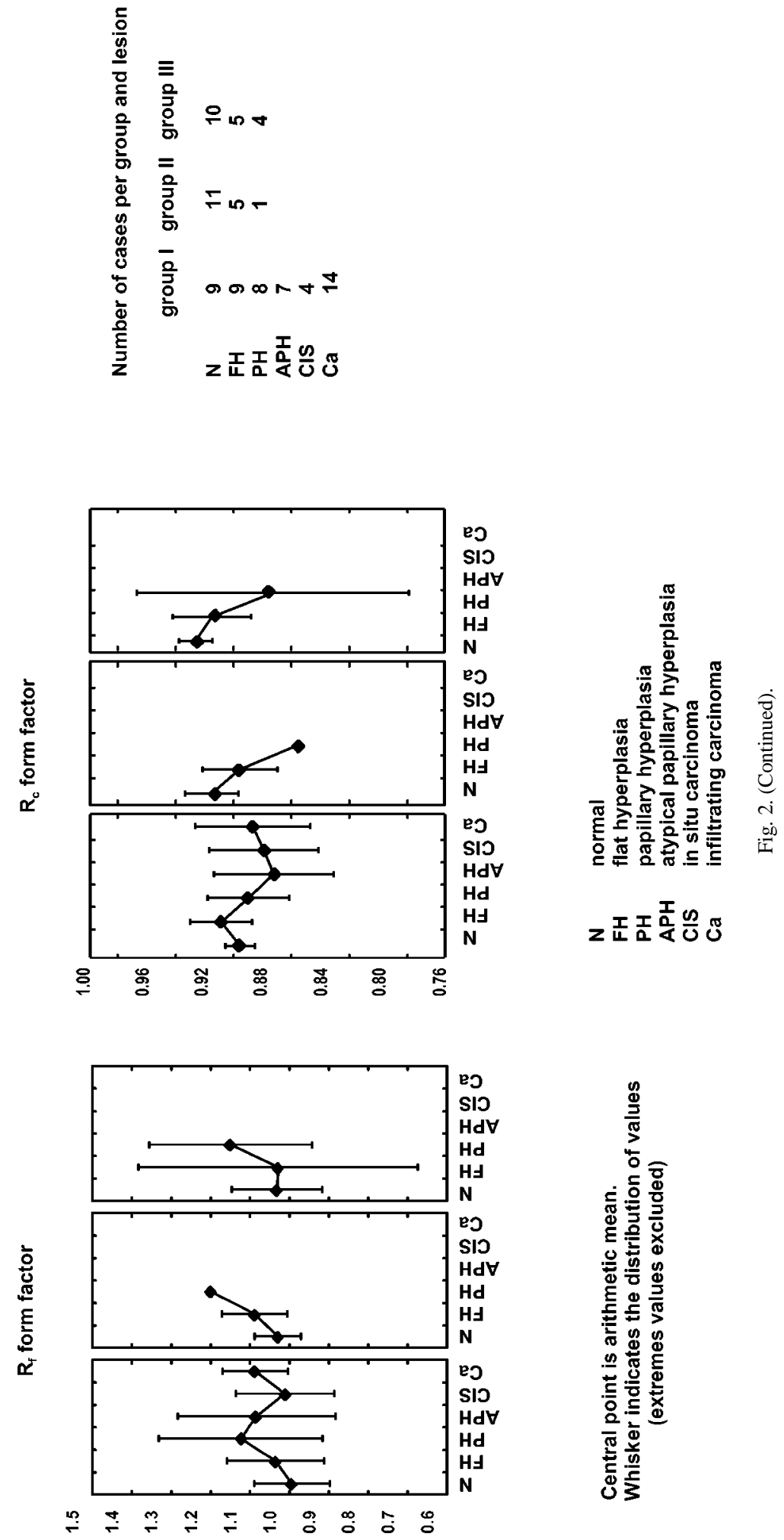
Table 1

Specific effects in group 1 (grouped into low and high grade lesions)

\begin{tabular}{|c|c|c|c|}
\hline & Low grade & vs. & High grade \\
\hline MIB-1 index & $U=19.00$ & & $p<0.001$ \\
\hline Area & $U=79.00$ & & $p<0.001$ \\
\hline Maximum diameter & $U=71.00$ & & $p<0.001$ \\
\hline Minimum diameter & $U=101.00$ & & $p<0.001$ \\
\hline SF & $U=211.00$ & & $\boldsymbol{p}<\mathbf{0 . 0 0 3}$ \\
\hline$R_{\mathrm{f}}$ & $U=380.00$ & & $p=0.51(\mathrm{~ns})$ \\
\hline$R_{\mathrm{c}}$ & $U=215.00$ & & $p=0.0032$ \\
\hline Elongation & $U=328.00$ & & $p=0.167(\mathrm{~ns})$ \\
\hline
\end{tabular}

$U$, Mann-Whitney statistics.

$p$, significance level, significant effects are marked in bold.

Low grade is normal, flat hyperplasia and papillary hyperplasia.

High grade is atypical papillary hyperplasia, in situ carcinoma and

infiltrating carcinoma.

Table 2

Correlation between Ki-67 expression index and selected geometric parameters

\begin{tabular}{lllllll}
\hline & \multicolumn{3}{c}{ Group 1 } & \multicolumn{2}{c}{ Group 2 } & \multicolumn{2}{c}{ Group 3 } \\
\hline Area & $\boldsymbol{R}=\mathbf{0 . 4 7}$ & $\boldsymbol{p}=\mathbf{0 . 0 1 9}$ & $\boldsymbol{R}=\mathbf{0 . 6 4}$ & $\boldsymbol{p}=\mathbf{0 . 0 1 7}$ & $\boldsymbol{R}=\mathbf{0 . 7 5}$ & $\boldsymbol{p}=\mathbf{0 . 0 0 1}$ \\
Max. diam. & $\boldsymbol{R}=\mathbf{0 . 5 2}$ & $\boldsymbol{p}=\mathbf{0 . 0 0 8}$ & $\boldsymbol{R}=\mathbf{0 . 6 5}$ & $\boldsymbol{p}=\mathbf{0 . 0 1 5}$ & $\boldsymbol{R}=\mathbf{0 . 7 1}$ & $\boldsymbol{p}=\mathbf{0 . 0 0 3}$ \\
Min. diam. & $R=0.38$ & $p=0.061$ & $R=0.40$ & $p=0.172$ & $\boldsymbol{R}=\mathbf{0 . 6 8}$ & $\boldsymbol{p}=\mathbf{0 . 0 0 5}$ \\
SF & $R=-0.31$ & $p=0.141$ & $R=-0.13$ & $p=0.662$ & $\boldsymbol{R}=-\mathbf{0 . 5 7}$ & $\boldsymbol{p}=\mathbf{0 . 0 2 6}$ \\
$\boldsymbol{R}_{\mathrm{f}}$ & $R=0.16$ & $p=0.458$ & $\boldsymbol{R}=\mathbf{0 . 7 0}$ & $\boldsymbol{p}=\mathbf{0 . 0 0 7}$ & $R=0.08$ & $p=0.790$ \\
$\boldsymbol{R}_{\mathrm{c}}$ & $R=0.29$ & $p=0.158$ & $R=-0.13$ & $p=0.661$ & $\boldsymbol{R}=\mathbf{0 . 5 5}$ & $\boldsymbol{p}=\mathbf{0 . 0 3 2}$ \\
Elongation & $R=-0.18$ & $p=0.395$ & $R=-0.38$ & $p=0.197$ & $R=-0.50$ & $p=0.057$ \\
\hline$R$, Spearman rank order correlation. & & & &
\end{tabular}

tissue storing probably does not affect the quality of immunohistochemical reaction $[4,12,42]$. Ki-67 index has been used in head and neck squamous epithelial carcinomas, where it has been found that in contrast to the normal state (where Ki-67 positive cells appear only in the reproductive area), in dysplasia and carcinoma in situ positive cells appear in all layers of the epithelium. With respect to neoplasms of the head and neck Ki-67 index correlated with $\mathrm{T}$ and $\mathrm{N}$ stage and histological grade [35]. Similar results were obtained in the study of cancer of the bronchus, urinary bladder and renal pelvis and larynx $[6,11,19,26]$. With respect to breast cancer it was found that $\mathrm{Ki}-67$ index is a very good marker of the proliferative activity and an effective indicator of prognosis $[24,31,41,43]$. In nonHodgkin lymphoma and in soft tissue sarcomas the number of Ki-67 positive cells showed a correlation with prognosis $[14,40]$. Healy in basal cell carcinomas of the skin found a relationship between the proliferation index and a tendency towards recurrences [15]. Pelosi studied Ki-67 immunoreactivity in endocrine tu- mours of the pancreas and demonstrated that it correlated with the tumor stage and may be used in the morphological assessment and therapy planning. According to Pelosi Ki-67 index was found to be better than PCNA or mitotic index both with respect to fresh and archival material [32]. The proliferative marker Ki-67 may be used to assess precancerous states from the viewpoint of risk for developing cancer. Hong in the study of Barrett's esophagus found out that with increasing degree of dysplasia the number of Ki-67 positive cells increased and the cells appeared in all layers of the epithelium, also on the surface where they replaced mature Ki-67 negative cells [16].

In view of the increasing frequency and poor prognosis of pancreatic cancer it is most important to identify morphological precancerous states, which may be detected with the available diagnostic methods, first of all, ultrasonography guided fine needle aspiration biopsy. In smears from pancreatic "tumours" one may find cells from intraductal proliferative lesions. The detection of normal and cancer elements is not difficult, 
whereas it is a problem to define the type of proliferation and to evaluate its possible tendency towards progression. To assess these changes we use classical cytological criteria, as there are no exact karyometric values for individual types of proliferation. For this reason in our study we made an attempt to calculate karyometric values such as the surface area, minimum, mean and maximum diameter and several form factors for individual types of pancreatic intraepithelial proliferative lesions. However, we are aware of the fact that these are not the criteria that may be extrapolated directly to routinely assessed cytological smears. In contrast, immunohistochemical analysis of the proliferative activity undoubtedly is suitable for use in differential diagnosis of problematic histological and cytological cases. In the present study we found out that in patients with pancreatic cancer in normal ductal epithelium a fraction of proliferating cells was $3.7 \%$, whereas in chronic pancreatitis the index was equal to zero, and in group III (extrapancreatic neoplasms) below $1 \%$. It may be thus concluded that in patients with pancreatic cancer those ductal epithelial cells, which in routine histological examination seem normal, may already have genetic alterations being a substrate for neoplasia. Lack of the proliferative activity in normal ductal epithelium in patients with chronic pancreatitis may be another argument in the discussion on the relationship between pancreatitis and the development of pancreatic cancer. It is necessary to comment on the degree of cell proliferation in intraepithelial proliferative lesions in group III. These patients were operated on for extrapancreatic neoplasms, therefore we should expect that pancreatic ductal epithelium is totally free of changes related to neoplasia. However, in this group of patients some of them were operated on for cancer of the papilla of Vater and it cannot be excluded that this neoplasm may have similar carcinogenic factors and genetic background to pancreatic cancer, which would result in higher than in chronic pancreatitis proliferative activity of pancreatic intraductal cells. This hypothesis is confirmed by the fact that in patients operated on for cancer of the papilla of Vater there was a tendency towards higher Ki-67 index than in patients operated on for gastric carcinoma or lymphoma. In patients operated on for pancreatic cancer we noted a gradual increase of the proliferative activity with increasing degree of intraepithelial proliferation where the highest values were found in infiltrating cancer. The demarcating line of statistical significance was between papillary hyperplasia and atypical papillary hyperplasia. This observation is an additional evidence in favor of the precancerous character of atypical papillary hyperplasia. It is also a basis for our classification of pancreatic intraepithelial proliferative lesions into two groups: low-grade and high-grade which we believe is better than using a number of not clearly defined categories. The low-grade group includes flat hyperplasia and papillary hyperplasia without atypia, whereas high-grade group- atypical papillary hyperplasia and carcinoma in situ due to the fact that differences in their proliferative activity do not show statistical significance. It should be stressed that papillary and flat hyperplasia differ in different study groups in relation to proliferation index and nuclear signatures. It means that lesions classified by light microscopy as flat and papillary hyperplasia may have different biology. This in turn may be related to the basic disease (pancreatic cancer or pancreatitis) and in pancreatic carcinoma-bearing patients may reflect early steps in carcinogenesis. We think that in histological classification of intraductal proliferative lesions diagnosis in based mostly at nuclear shape whereas other nuclear features seem to be less important. It should be noted that none of the individual parameters permits effective differentiation among proliferative lesions. These findings are compatible with the idea of using a set of features (nuclear signatures) instead of single ones [3].

The values of Ki-67 index in the present study are generally in accordance with the data obtained by Nagai. He demonstrated that the mean proliferation index in hyperplastic epithelium (he does not describe in detail the type of proliferation) is $4.6 \%$, in moderate atypical adenomatous epithelium $16.9 \%$, in carcinoma in situ $39.8 \%$, and in infiltrating cancer $47.4 \%$. The index closely correlated with types of proliferation and was significantly higher in cancer than in hyperplasia or adenomatous epithelium [28]. Terada found the high proliferative activity of pancreatic ductal carcinoma cells in contrast to intraductal papillary mucinous neoplasms of the pancreas. In his results similar to our study the proliferative activity increased with the degree of dysplasia, which confirms the usefulness of $\mathrm{Ki}-67$ index in evaluating the risk of progression of pancreatic intraepithelial proliferative lesions [38]. From the practical viewpoint it should be assumed that Ki-67 index below $15 \%$ indicates proliferation of lowgrade type. This information together with the cytological features of proliferating cells of pancreatic ductal epithelium serve as a guideline in planning the frequency of control studies in the patient. The patients in whom the proliferation index in intraepithelial proliferation areas would be over $15 \%$ should be classi- 
fied into a group at increased risk and depending on the severity of proliferative lesions undergo more frequent controls or be selected for surgical treatment.

\section{Conclusions}

1. There are two distinct groups of pancreatic intraductal proliferative lesions: low-grade and highgrade, and the demarcating line goes between papillary hyperplasia and atypical papillary hyperplasia.

2. Low-grade lesions in individual study groups differ with respect to the proliferative activity and karyometric data, and these differences are probably related to the underlying disease.

3. The proliferation index $\mathrm{Ki}-67$ is a good marker differentiating between low-grade and high-grade proliferations.

4. Differences in the proliferation index $\mathrm{Ki}-67$ in atypical papillary hyperplasia and in carcinomas in situ are not significant, which indicates that malignancy does not mean exclusively increased cell proliferation.

5. A set of karyometric features in pancreatic intraductal proliferative lesions correlates with the proliferation index $\mathrm{Ki}-67$, whereas there is no single feature that would have a prognostic value.

\section{Acknowledgement}

This study was supported by Grant KBN (number 4PO5C04413), and Jagiellonian University Program (number WL C/P/135/98).

\section{References}

[1] AnalySIS imaging C, Soft-Imaging Software GmbH, Münster, Germany, 1996.

[2] AnalySIS User Guide, Soft-Imaging Software GmbH, Münster, Germany, 1996.

[3] P. Bartels, Automation in the objective analysis of prostate lesions, in: EuroCellPath '98. Molecular and Quantitative Analysis of Early Neoplasia, Belfast, UK, 1998.

[4] A.M. Bilous, M. McKay and J. Milliken, A comparison between Ki-67 antibody reactivity and other pathological variables in breast carcinoma, Pathology 23 (1991), 282-285.

[5] G. Cattoretti, M.H.G. Becker, G. Key, M. Duchrow, C. Schluter, J. Galle and J. Gerdes, Monoclonal antibodies against recombinant parts of the Ki-67 antigen (MIB1 and MIB3) detect proliferating cells in microwave-processed formalin-fixed paraffin sections, J. Pathol. 168 (1992), 357-363.
[6] J.C. Cheville, R.B. Terrell and M.B. Cohen, Use of proliferative markers Ki-67 (MIB-1) and proliferating cell nuclear antigen (PC10) in transitional cell carcinoma of the renal pelvis, Mod. Pathol. 7 (1994), 794-800.

[7] A.L. Cubilla and P.J. Fitzgerald, Morphological lesions associated with human primary invasive nonendocrine pancreas cancer, Cancer Res. 36 (1976), 2690-2698.

[8] J.D. Day, J.A. DiGiuseppe, C. Yeo, M. Lai-Goldman, S. Andreson, S.N. Goodman, S.E. Kern and R.H. Hruban, Immunohistochemical evaluation of HER-2/neu expression in pancreatic adenocarcinoma and pancreatic intraepithelial neoplasms, Hum. Pathol. 27 (1996), 119-124.

[9] J. Gerdes, H. Lemke, H. Baisch, H.-H. Wacker, U. Schwab and H. Stein, Cell cycle analysis of a cell proliferation-associated human nuclear antigen defined by the monolconal antibody Ki67, J. Immunol. 133 (1984), 1710-1715.

[10] J. Gerdes, L. Li, C. Schlueter, M. Duchrow, C. Wohlenberg, C. Gerlach, I. Stahmer, S. Kloth, E. Brandt and H.-D. Flad, Immunobiochemical and molecular biologic characterization of the cell proliferation-associated nuclear antigen that is defined by monoclonal antibody Ki-67, Am. J. Pathol. 138 (1991), 867873.

[11] W. Golusiński, J. Olofsson, Z. Szmeja and P. Majewski, p53, PCNA and Ki-67 in laryngeal cancer, Pol. J. Pathol. 4 (1996), 175-182.

[12] P.A. Hall and P.J. Coates, Assessment of cell proliferation in pathology - what next?, Histopathology 26 (1995), 105-112.

[13] P.A. Hall, C.M. Hughes, S.L. Staddon, P.L. Richman, W.J. Gullick and N.R. Lemoine, The c-erbB-2 protooncogene in human pancreatic carcinoma, J. Pathol. 161 (1990), 195-222.

[14] P.A. Hall, M.A. Richards, W.M. Gregory, A.J. d'Ardenne, T.A. Lister and A.G. Stansfeld, The prognostic value of Ki67 immunostaining in non-Hodgkin lymphoma, J. Pathol. 154 (1988), 223-235.

[15] E. Healy, B. Angus, C.M. Lawrence and J.L. Rees, Prognostic value of Ki-67 antigen expression in basal cell carcinomas, $\mathrm{Br}$. J. Dermatol. 133 (1995), 737-741.

[16] M.K. Hong, W.B. Laskin, B.E. Herman, M.H. Johnston, J.J. Vargo, S.M. Steinberg, C.J. Allegra and P.G. Johnston, Expansion of the Ki-67 proliferative compartment correlates with degree of dysplasia in Barrett's esophagus, Cancer 75 (1995), 423-429.

[17] M. Kaino, Alterations in the tumor suppressor genes p53, RB, p16/MTS1 and p15/MTS2 in human pancreatic cancer and hepatoma cell lines, J. Gastroenterol. 32 (1997), 40-46.

[18] H. Kalthoff, W. Schmiegel, C. Roeder, D. Kasche, A. Schmidt, G. Lauer, H.-G. Thiele, G. Honold, K. Pantel, G. Riethmuller, E. Scherer, J. Maurer, H. Maacke and W. Deppert, p53 and KRAS alterations in pancreatic epithelial cell lesions, Oncogene 8 (1993), 289-298.

[19] T. Kawai, M. Suzuki, S. Kono, S. Kono, N. Shinomiga, M. Rokutanda, K. Takagi, T. Ogata and S. Tamai, Proliferating cell nuclear antigen and Ki-67 in lung carcinoma. Correlation with DNA flow cytometric analysis, Cancer 74 (1994), 2468-2475.

[20] G. Kloppel, Gene changes and pancreatic carcinoma. The significance of K-ras, Dig. Surg. 1 (1994), 164-169.

[21] S. Kozuka, R. Sassa, T. Taki, K. Masamoto, S. Nagasawa, S. Saga, K. Hasegawa and M. Takeuchi, Relation of pancreatic duct hyperplasia to carcinoma, Cancer 43 (1979), 1418-1428. 
[22] N.R. Lemoine, S. Jain, C.M. Hughes, S.L. Staddon, B. Maillet, P.A. Hall and G. Kloppel, Ki-ras oncogene activation in preinvasive pancreatic cancer, Gastroenterology 102 (1992), 230236.

[23] A. Łomnicki, Wprowadzenie do Statystyki dla Przyrodników, Wydawnictwo Naukowe PWN, Warszawa, 1995.

[24] M. Markiewski and W. Domagała, Immunohistochemical assessment of proliferation rate of breast carcinoma cells using Ki-67, MIB-1 and anti-PCNA monoclonal antibodies, Pol. J. Pathol. 4 (1996), 189-194.

[25] D. McCormick, H. Chong, C. Hobbs, C. Datta and P.A. Hall, Detection of the Ki-67 antigen in fixed and wax-embedded sections with the monoclonal antibody MIB1, Histopathology 22 (1993), 355-360.

[26] A.H. Mulder, J.C. Van Hootegem, R. Sylvester, F.J.W. Ten Kate, K.H. Kurth, E.C.M. Ooms and Th.H. Van Der Kwast, Prognostic factors in bladder carcinoma: histologic parameters and expression of a cell cycle-related nuclear antigen (Ki-67), J. Pathol. 166 (1992), 37-43.

[27] H.R. Myler and A.R. Weeks, The Pocket Handbook of Image Processing Algorithms in C, Prentice-Hall, Eglewood Cliffs, NJ, USA, 1993.

[28] E. Nagai, T. Ueki, K. Chijiiwa, M. Tanaka and M. Tsuneyoshi, Intraductal papillary mucinous neoplasms of the pancreas associated with so-called "mucinous ductal ectasia", Am. J. Surg. Pathol. 19 (1995), 576-589.

[29] K. Okoń, A. Basta and J. Stachura, Morphometry separates squamous cell carcinoma of the vulva into two distinct groups, Pol. J. Pathol. 49 (1998), 293-295.

[30] J.R. Parker, Algorithms for Image Processing and Computer Vision, John Wiley \& Sons, New York, Chichester, Brisbane, Toronto, Singapore, Weinheim, 1997.

[31] Z.P. Pavelic, L. Pavelic, E.E. Lower, M. Gapany, S. Gapany, E.A. Barker and H.D. Preisler, C-myc, c-erbB-2, and Ki-67 expression in normal breast tissue and in invasive and noninvasive breast carcinoma, Cancer Res. 52 (1992), 2597-2602.

[32] G. Pelosi, E. Bresaola, G. Bogina, F. Pasini, S. Rodella, P. Castelli, C. Iacono, G. Serio and G. Zamboni, Endocrine tumors of the pancreas: Ki-67 immunoreactivity on paraffin sections is an independent predictor for malignancy, Hum. Pathol. 27 (1996), 1124-1134.

[33] A. Ragen, Leksykon Jezyka C, Wydawnictwo NaukowoTechniczne, Warszawa, 1990.
[34] R.J. Shalkoff, Digital Image Processing and Computer Vision, John Wiley \& Sons, New York, Chichester, Brisbane, Toronto, Singapore, 1989.

[35] P.J. Slootweg, R. Koole and G.T. Hordijk, The presence of p53 protein in relation to $\mathrm{Ki} 67$ as cellular proliferation marker in head and neck squamous cell carcinoma and adjacent dysplastic mucosa, Oral Oncol. Eur. J. Cancer 30B (1994), 138-141.

[36] A. Stanisz, Przystępny kurs statystyki w oparciu o program STATISTICA PL na przykładach z medycyny, StatSoft Polska, 1998.

[37] R. Tadeuszewicz and P. Korohoda, Komputerowa analiza i przetwarzanie obrazów, Wydawnictwo Fundacji Postępu Telekomunikacji, Kraków, 1997.

[38] T. Terada, T. Ohta, Y. Kitamura, K. Ashida and Y. Matsunaga, Cell proliferative activity in intraductal papillary-mucinous neoplasms and invasive ductal adenocarcinomas of the pancreas: an immunohistochemical study, Arch. Pathol. Lab. Med. 122 (1998), 42-46.

[39] R. Tomaszewska, K. Okoń, K. Nowak and J. Stachura, HER$2 /$ neu expression as a progression marker in pancreatic intraepithelial neoplasia, Pol. J. Pathol. 49 (1998), 83-92.

[40] T. Ueda, K. Aozasa, M. Tsujimoto, M. Ohsawa, A. Uchida, Y. Aoki, K. Ono and K. Matsumoto, Prognostic significance of Ki-67 reactivity in soft tissue sarcomas, Cancer 63 (1989), 1607-1611.

[41] S.M. Veronese, M. Gambacorta, O. Gottardi, F. Scanzi, M. Ferrari and P. Lampertico, Proliferation index as a prognostic marker in breast cancer, Cancer 71 (1993), 3926-3931.

[42] N. Weidner, D.H. Moore and R. Vartanian, Correlation of Ki-67 antigen expression with mitotic figure index and tumor grade in breast carcinomas using the novel "paraffin"-reactive MIB 1 antibody, Hum. Pathol. 25 (1994), 337-342.

[43] H.-O. Wintzer, I. Zipfel, J. Schulte-Monting, U. Hellerich and S. von Kleist, Ki-67 immunostaining in human breast tumors and its relationship to prognosis, Cancer 67 (1991), 421-428.

[44] J.T. Woosley, Measuring cell proliferation, Arch. Pathol. Lab. Med. 115 (1991), 555-557.

[45] K.W. Zieliński, Zarys zasad histomorfometrii w badaniach patomorfologicznych, Pol. J. Pathol. 45(4) (1994). 


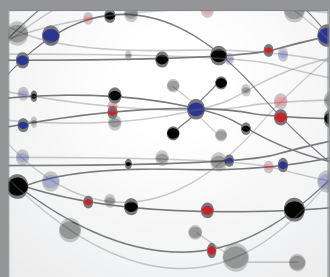

The Scientific World Journal
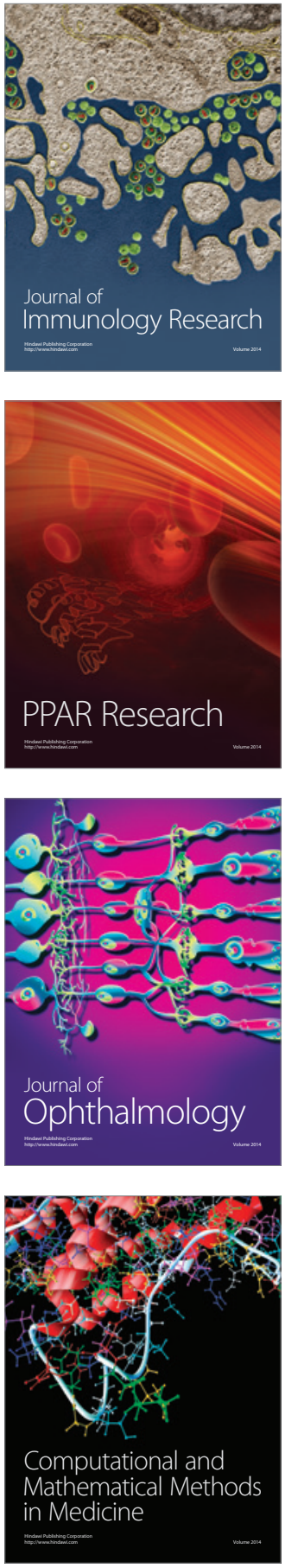

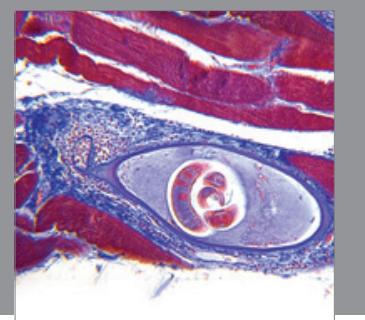

Gastroenterology

Research and Practice
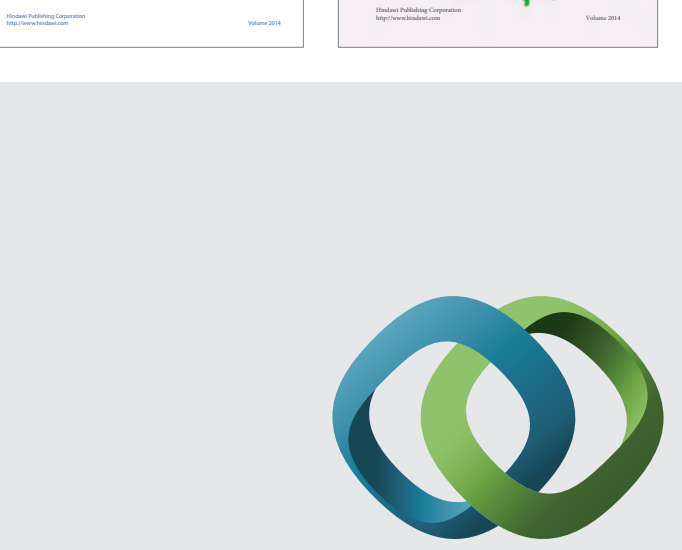

\section{Hindawi}

Submit your manuscripts at

http://www.hindawi.com
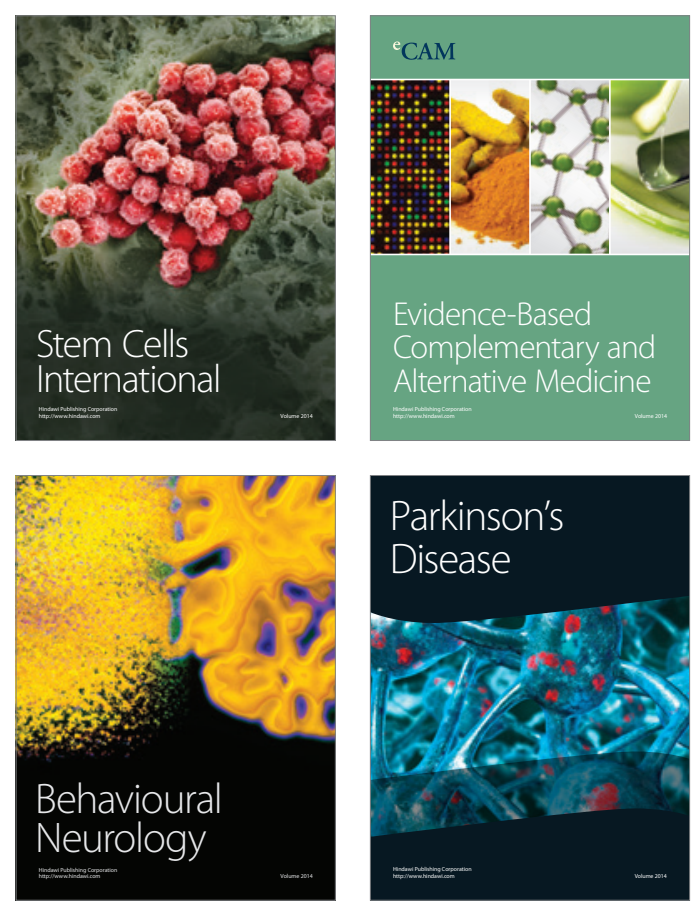

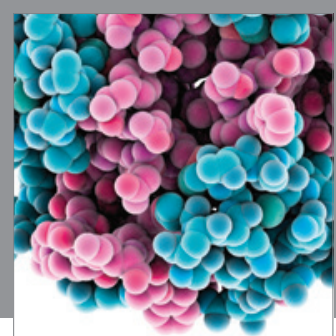

Journal of
Diabetes Research

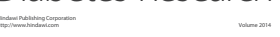

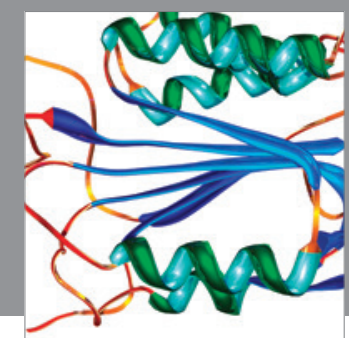

Disease Markers
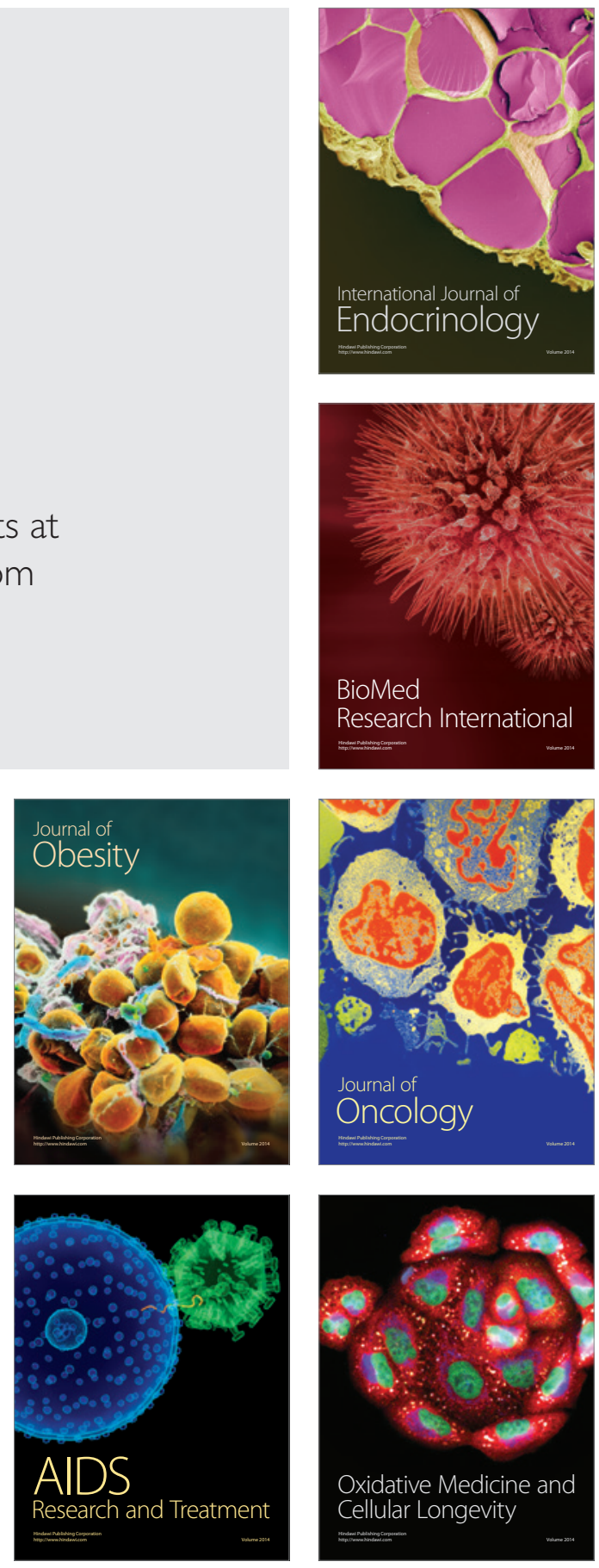